\title{
Solubility of Pyrene in Binary Alcohol + 2-Methyl-2-butanol Solvent Mixtures at $299.2 \mathrm{~K}$
}

\author{
Mary E. R. McHale, Karen S. Coym, Kristin A. Fletcher, and William E. Acree, J r.* \\ Department of Chemistry, University of North Texas, Denton, Texas 76203-0068
}

\begin{abstract}
Experimental solubilities are reported for pyrene dissolved in eight binary mixtures containing 2-methyl2-butanol and alcohol (1-propanol, 2-propanol, 1-butanol, 2-methyl-1-propanol, 1-pentanol, 2-pentanol, 3-methyl-1-butanol, or 4-methyl-2-pentanol) at $26{ }^{\circ} \mathrm{C}$. Results of these measurements are used to test two mathematical representations based upon the combined nearly ideal binary solvent (NIBS)/RedlichKister and modified Wilson equations. For the eight systems studied, both equations were found to provide an accurate mathematical representation of the experimental data, with an overall average absolute deviation between measured and calculated values being $0.3 \%$ and $0.4 \%$ for the combined NIBS/RedlichKister and modified Wilson equations, respectively. Also reported are experimental solubilities for pyrene dissolved in 2-methoxyethanol, 2-ethoxyethanol, 2-propoxyethanol, 2-butoxyethanol, and 3-methoxy-1butanol.
\end{abstract}

\section{Introduction}

Solid-liquid equilibrium data of organic nonelectrolyte systems are becoming increasingly important in the petroleum industry, particularly in light of present trends toward heavier feedstocks and known carcinogenicity/ mutagenicity of many of the larger polycyclic aromatic compounds. Solubility data for a number of polycyclic aromatic hydrocarbons (i.e, anthracene and pyrene) and heteroatom polynuclear aromatics (i.e., carbazole, dibenzothiophene, and xanthene) have been published in the recent chemical literature (for listing of references see Acree, 1994, 1995a,b). Despite efforts by experimentalists and scientific organizations, in terms of both new experimental measurements and critically-evaluated data compilations, there still exist numerous systems for which solubility data are not readily available.

To address this problem, researchers have turned to predictive methods as a means to generate desired quantities. Numerous equations have been suggested for predicting solute solubilities in binary solvent mixtures. For the most part, the predictive methods do provide fairly reasonable estimates for noncomplexing systems. There still remains, however, the need to develop better predictive expressions and mixing models to describe the more nonideal complexing systems believed to contain hydrogenbonding solvent components. Continued development of solution models for describing the thermodynamic properties of a solute in binary solvent systems requires that a large data base be available for assessing the applications and limitations of derived expressions. Currently, only a limited data base exists for crystalline nonelectrolyte solubility in binary solvent mixtures capable of undergoing hydrogen-bond formation. For this reason, pyrene solubilities were determined in eight binary alcohol + 2-methyl2-butanol solvent mixtures. Results of these measurements are used to further test the descriptive abilities of two previously derived expressions. Also reported are experimental solubilities for pyrene dissolved in five alkoxy alcohols.

* To whom correspondence should be addressed. E-mail: acree@cas1.unt.edu.

\section{Experimental Methods}

Pyrene (Aldrich, 99\%) was recrystallized several times from anhydrous methanol, giving a purified sample that melted at $\mathrm{T}_{\mathrm{mp}} / \mathrm{K}=426.2 \pm 0.5$. 2-Methyl-2-butanol (Acros, 99\%), 1-propanol (Aldrich 99+\%, anhydrous), 2-propanol (Aldrich 99+\%, anhydrous), 1-butanol (Aldrich HPLC, 99.8+\%), 2-methyl-1-propanol (Aldrich 99+\%, anhydrous), 1-pentanol (Aldrich 99\%), 2-pentanol (Acros, 99+\%), 3-methyl-1-butanol (Aldrich, 99+\%, anhydrous), 4-methyl-2-pentanol (Acros 99+\%), 2-methoxyethanol (Aldrich, 99.5+\%, anhydrous), 2-ethoxyethanol (Acros, 99\%), 2-propoxyethanol (Aldrich, 99+\%), 2-butoxyethanol (Acros, 99\%), and 3-methoxy-1-butanol (Aldrich, 99+\%) were stored over both anhydrous sodium sulfate and mol ecular sieves before use. Gas chromatographic analysis showed solvent purities to be 99.7 mol \% or better. Karl Fischer titration gave water contents (mass $/$ mass $\%$ ) of $<0.01 \%$ for all eight al cohols and five alkoxy alcohols used. Binary solvent mixtures were prepared by mass so that compositions could be calculated to 0.0001 mole fraction.

Excess solute and solvent were placed in amber glass bottles and allowed to equilibrate in a constant temperature water bath at $(26.0 \pm 0.1){ }^{\circ} \mathrm{C}$ with periodic agitation for at least 3 days (often longer). Attainment of equilibrium was verified both by repetitive measurements after a minimum of three additional days and by approaching equilibrium from supersaturation by pre-equilibrating the solutions at a higher temperature. Aliquots of saturated pyrene solutions were transferred through a coarse filter into a tared volumetric flask to determine the amount of sample and diluted quantitatively with methanol for spectrophotometric analysis at $372 \mathrm{~nm}$ on a Bausch and Lomb Spectronic 2000. Concentrations of the dilute solutions were determined from a Beer-Lambert law absorbance versus concentration working curve derived from measured absorbances of standard solutions of known molar concentration. Molar absorptivities of the nine standard solutions varied systematically with molar concentration and ranged from approximately $\epsilon / \mathrm{L} \mathrm{mol}^{-1} \mathrm{~cm}^{-1}=234$ to $\epsilon / \mathrm{L} \mathrm{mol}^{-1} \mathrm{~cm}^{-1}$ $=220$ for pyrene concentrations ranging from $\mathrm{C} / \mathrm{mol} \mathrm{L}^{-1}$ $=8.31 \times 10^{-4}$ to $\mathrm{C} / \mathrm{mol} \mathrm{L}^{-1}=4.15 \times 10^{-3}$. I dentical molar absorptivities were obtained for select pyrene standard solutions that contained up to 5 volume $\%$ of the neat alcohol and alkoxy alcohol cosolvents. Experimental pyrene 
Table 1. Experimental Mole Fraction Solubilities of Pyrene $\left(x_{A}^{\text {sat }}\right)$ in Binary Alcohol (B) + 2-Methyl-2-butanol (C) Solvent Mixtures at $26.0^{\circ} \mathrm{C}$

\begin{tabular}{|c|c|c|c|}
\hline$x_{C}^{\circ}$ & $x_{A}^{\text {sat }}$ & $x_{C}^{\circ}$ & $x_{A}^{\text {sat }}$ \\
\hline \multicolumn{4}{|c|}{ 1-Propanol (B) + 2-M ethyl-2-butanol (C) } \\
\hline 0.0000 & 0.00426 & 0.5113 & 0.00507 \\
\hline 0.0774 & 0.00435 & 0.7306 & 0.00552 \\
\hline 0.1462 & 0.00442 & 0.8507 & 0.00579 \\
\hline 0.3084 & 0.00469 & 1.0000 & 0.00617 \\
\hline 0.4092 & 0.00491 & & \\
\hline \multicolumn{4}{|c|}{ 2-Propanol (B) + 2-Methyl-2-butanol (C) } \\
\hline 0.0000 & 0.00290 & 0.5082 & 0.00442 \\
\hline 0.0845 & 0.00311 & 0.7240 & 0.00512 \\
\hline 0.1468 & 0.00330 & 0.8588 & 0.00560 \\
\hline 0.3101 & 0.00375 & 1.0000 & 0.00617 \\
\hline 0.4046 & 0.00405 & & \\
\hline \multicolumn{4}{|c|}{ 1-Butanol (B) + 2-Methyl-2-butanol (C) } \\
\hline 0.0000 & 0.00622 & 0.5603 & 0.00620 \\
\hline 0.0911 & 0.00618 & 0.7567 & 0.00617 \\
\hline 0.1812 & 0.00616 & 0.8725 & 0.00619 \\
\hline 0.3587 & 0.00620 & 1.0000 & 0.00617 \\
\hline 0.4356 & 0.00618 & & \\
\hline \multicolumn{4}{|c|}{ 2-Methyl-1-propanol (B) + 2-Methyl-2-butanol (C) } \\
\hline 0.0000 & 0.00326 & 0.5696 & 0.00463 \\
\hline 0.0970 & 0.00342 & 0.7668 & 0.00528 \\
\hline 0.1808 & 0.00363 & 0.8703 & 0.00570 \\
\hline 0.3659 & 0.00408 & 1.0000 & 0.00617 \\
\hline 0.4755 & 0.00441 & & \\
\hline \multicolumn{4}{|c|}{ 1-Pentanol (B) + 2-M ethyl-2-butanol (C) } \\
\hline 0.0000 & 0.00931 & 0.5989 & 0.00756 \\
\hline 0.1101 & 0.00913 & 0.7969 & 0.00684 \\
\hline 0.1955 & 0.00885 & 0.8883 & 0.00656 \\
\hline 0.3939 & 0.00818 & 1.0000 & 0.00617 \\
\hline 0.4866 & 0.00777 & & \\
\hline \multicolumn{4}{|c|}{ 2-Pentanol (B) + 2-M ethyl-2-butanol (C) } \\
\hline 0.0000 & 0.00640 & 0.6077 & 0.00622 \\
\hline 0.0997 & 0.00638 & 0.8021 & 0.00620 \\
\hline 0.2066 & 0.00634 & 0.8949 & 0.00621 \\
\hline 0.3816 & 0.00629 & 1.0000 & 0.00617 \\
\hline 0.5101 & 0.00626 & & \\
\hline \multicolumn{4}{|c|}{ 3-Methyl-1-butanol (B) + 2-M ethyl-2-butanol (C) } \\
\hline 0.0000 & 0.00546 & 0.5912 & 0.00587 \\
\hline 0.1276 & 0.00558 & 0.7903 & 0.00598 \\
\hline 0.2134 & 0.00563 & 0.8931 & 0.00606 \\
\hline 0.3988 & 0.00572 & 1.0000 & 0.00617 \\
\hline 0.4997 & 0.00581 & & \\
\hline \multicolumn{4}{|c|}{ 4-Methyl-2-pentanol (B) + 2-M ethyl-2-butanol (C) } \\
\hline 0.0000 & 0.00621 & 0.6416 & 0.00626 \\
\hline 0.1324 & 0.00623 & 0.8168 & 0.00626 \\
\hline 0.2280 & 0.00626 & 0.9070 & 0.00621 \\
\hline 0.4365 & 0.00628 & 1.0000 & 0.00617 \\
\hline 0.5368 & 0.00629 & & \\
\hline
\end{tabular}

Table 2. Experimental Mole Fraction Solubilities of Pyrene $\left(x_{A}^{\text {sat }}\right)$ in Neat Alkoxy Alcohols at $26.0^{\circ} \mathrm{C}$

\begin{tabular}{lc}
\hline alkoxy alcohol solvent & $\mathrm{x}_{\mathrm{A}}^{\text {sat }}$ \\
\hline 2-methoxyethanol & 0.01717 \\
2-ethoxyethanol & 0.03046 \\
2-propoxyethanol & 0.03400 \\
2-butoxyethanol & 0.03790 \\
3-methoxy-1-butanol & 0.02541
\end{tabular}

solubilities in the eight binary alcohol +2 -methyl-2-butanol mixtures and in the five neat alkoxy alcohols studied are listed in Tables 1 and 2, respectively. Numerical values represent the average of between four and eight independent determinations, with the measured values being reproducible to within $\pm 1.3 \%$.

\section{Results and Discussion}

Acree and co-workers (Acree and Zvaigzne, 1991) suggested possible mathematical representations for isother-
Table 3. Mathematical Representation of Pyrene Solubilities in Several Binary Alcohol (B) + 2-Methyl-2-Butanol (C) Solvent Mixtures

\begin{tabular}{|c|c|c|c|c|}
\hline \multirow[b]{2}{*}{$\begin{array}{l}\text { binary solvent system component } \\
\text { (B) + component (C) }\end{array}$} & \multicolumn{2}{|c|}{ eq 1} & \multicolumn{2}{|c|}{ eq 2} \\
\hline & $\mathrm{Si}^{\mathrm{a}}$ & $\begin{array}{c}\% \\
\operatorname{dev}^{b}\end{array}$ & $\Lambda_{\mathrm{ij}}^{\mathrm{adj} c}$ & $\begin{array}{c}\% \\
\operatorname{dev}^{b}\end{array}$ \\
\hline 1-propanol + 2-methyl-2-butanol & -0.079 & 0.4 & $\begin{array}{l}1.347 \\
0.680\end{array}$ & 0.3 \\
\hline 2-propanol +2 -methyl-2-butanol & $\begin{array}{r}0.131 \\
0.019 \\
-0.069\end{array}$ & 0.3 & $\begin{array}{l}1.115 \\
0.912\end{array}$ & 0.3 \\
\hline 1-butanol + 2-methyl-2-butanola & & 0.3 & $\begin{array}{l}2.768 \\
0.361\end{array}$ & 0.3 \\
\hline $\begin{array}{c}\text { 2-methyl-1-propanol + } \\
\text { 2-methyl-2-butanol }\end{array}$ & $\begin{array}{l}-0.026 \\
-0.073 \\
-0.056\end{array}$ & 0.5 & $\begin{array}{l}1.173 \\
0.796\end{array}$ & 0.4 \\
\hline 1-pentanol +2 -methyl-2-butanol & $\begin{array}{l}0.115 \\
0.058 \\
0.145\end{array}$ & 0.4 & $\begin{array}{l}1.463 \\
0.796\end{array}$ & 0.6 \\
\hline 2-pentanol +2 -methyl-2-butanol & -0.013 & 0.1 & $\begin{array}{l}2.594 \\
0.390\end{array}$ & 0.1 \\
\hline $\begin{array}{l}\text { 3-methyl-1-butanol }+ \\
\text { 2-methyl-2-butanola }\end{array}$ & & 0.4 & $\begin{array}{l}1.028 \\
0.970\end{array}$ & 0.4 \\
\hline $\begin{array}{l}\text { 4-methyl-2-pentanol + } \\
\text { 2-methyl-2-butanol }\end{array}$ & 0.062 & 0.2 & $\begin{array}{l}0.651 \\
1.579\end{array}$ & 0.2 \\
\hline
\end{tabular}

a Combined NIBS/Redlich-Kister curve-fit parameters are ordered as $\mathrm{S}_{0}, \mathrm{~S}_{1}$, and $\mathrm{S}_{2}$. No curve-fit parameters were required in the case of the 1-butanol +2 -methyl-2-butanol and 3-methyl1-butanol + 2-methyl-2-butanol systems. ${ }^{b}$ Deviation (\%) $=(100 /$ 7) $\sum\left|\left[\left(x_{A}^{\text {sat }}\right)^{\text {calc }}-\left(x_{A}^{\text {sat }}\right)^{\exp }\right] /\left(x_{A}^{\text {sat }}\right)^{\exp }\right| .{ }^{c}$ Adjustable parameters for the modified Wilson equation are ordered as $\Lambda_{\mathrm{BC}}^{\text {adj }}$ and $\Lambda_{\mathrm{CB}}^{\text {adj }}$.

mal solubility data based upon either a combined NIBS/ Redlich-Kister model

$$
\ln x_{A}^{s a t}=x_{B}^{\circ} \ln \left(x_{A}^{s a t}\right)_{B}+x_{C}^{\circ} \ln \left(x_{A}^{s a t}\right)_{C}+x_{B}^{\circ} x_{C}^{\circ} \sum_{i=0}^{N} S_{i}\left(x_{B}^{\circ}-x_{C}^{\circ}\right)^{i}
$$

or modified Wilson equation

$$
\begin{aligned}
& \ln \left[\mathrm{a}_{\mathrm{A}}(\mathrm{s}) / \mathrm{x}_{\mathrm{A}}^{\mathrm{sat}}\right]=1-\mathrm{x}_{\mathrm{B}}^{\circ}\left\{1-\ln \left[\mathrm{a}_{\mathrm{A}}(\mathrm{s}) /\left(\mathrm{x}_{\mathrm{A}}^{\mathrm{sat}}\right)_{\mathrm{B}}\right]\right\} /\left(\mathrm{x}_{\mathrm{B}}^{\circ}+\right. \\
& \left.\mathrm{x}_{\mathrm{C}}^{\circ} \Lambda_{\mathrm{BC}}^{\mathrm{adj}}\right)-\mathrm{x}_{\mathrm{C}}^{\circ}\left\{1-\ln \left[\mathrm{a}_{\mathrm{A}}(\mathrm{s}) /\left(\mathrm{x}_{\mathrm{A}}^{\mathrm{sat}}\right)_{\mathrm{C}}\right]\right\} /\left(\mathrm{x}_{\mathrm{B}}^{\circ} \Lambda_{\mathrm{CB}}^{\mathrm{adj}}+\mathrm{x}_{\mathrm{C}}^{\circ}\right)
\end{aligned}
$$

where the various $S_{i}$ and $\Lambda_{i j}^{\text {adj }}$ "curve-fit" parameters can be evaluated via least squares analysis. In eqs 1 and $2 x_{B}^{\circ}$ and $x_{C}^{\circ}$ refer to the initial mole fraction composition of the binary solvent calculated as if solute $(A)$ were not present, $a_{A}(s)$ is the activity of the solid solute, $N$ is the number of curve-fit parameters used, and $\left(x_{A}^{s a t}\right)_{i}$ is the saturated mole fraction solubility of the solute in pure solvent $i$. The numerical value of $a_{A}(s)$ used in the modified Wilson computations was $a_{A}(s)=0.1312$ (J udy et al., 1987).

The ability of eqs 1 and 2 to mathematically represent the experimental solubility of pyrene in the eight binary alcohol + 2-methyl-2-butanol solvent systems is summarized in Table 3 in the form of "curve-fit" parameters and percent deviations in back-calculated solubilities. Careful examination of Table 3 reveals that both equations provide an accurate mathematical representation for how the solubility of pyrene varies with solvent composition. For the eight pyrene systems studied, the overall average absolute deviation between the experimental and calculated values is $0.3 \%$ and $0.4 \%$ for eqs 1 and 2 , respectively, which is less than the experimental uncertainty.

For informational purposes, it is noted that during the past 3 years we (McHale et al., 1996a,b) have developed expressions for estimating polycyclic aromatic hydrocarbon solubilities in binary alcohol + alcohol and alcohol + alkoxy 
alcohol solvent mixtures based upon the mobile order theory and the Kretschmer-Wiebe association model. Predictive equations derived from both the mobile order theory and the K retschmer-Wiebe model require as input values measured solute solubilities in both pure solvents. Several other published predictive methods have similar input requirements. Mole fraction solubilities listed in Table 2 for pyrene dissolved in 2-methoxyethanol, 2-ethoxyethanol, 2-propoxyethanol, 2-butoxyethanol, and 3-methoxy-1-butanol are reported in order to facilitate estimation of pyrene solubilities in binary alcohol + alkoxy alcohol mixtures.

\section{Literature Cited}

(1) Acree, W. E., J r. Polycyclic Aromatic Hydrocarbons in Pure and Binary Solvents; Vol. 54 in IUPAC Solubility Data Series; Oxford University Press: Oxford, United Kingdom, 1994.

(2) Acree, W. E., J r. Polycyclic Aromatic Hydrocarbons: Binary Nonaqueous Systems: Part 1 (Solutes A-E); Volume 58 in IUPAC Solubility Data Series; Oxford University Press: Oxford, United Kingdom, 1995a.

(3) Acree, W. E., J r. Polycyclic Aromatic Hydrocarbons: Binary Nonaqueous Systems: Part 2 (Solutes F-Z); Volume 59 in IUPAC Solubility Data Series; Oxford University Press: Oxford, United Kingdom, 1995b.
(4) Acree, W. E., J r.; Zvaigzne, A. I. Thermodynamic Properties of Nonelectrolyte Solutions. Part 4. Estimation and Mathematical Representation of Solute Activity Coefficients and Solubilities in Binary Solvents Using the NIBS and Modified Wilson Equations. Thermochim. Acta 1991, 178, 151-167.

(5) J udy, C. L.; Pontikos, N. M.; Acree, W. E., J r. Solubility of Pyrene in Binary Solvent Mixtures Containing Cyclohexane. J . Chem. Eng. Data 1987, 32, 60-62.

(6) McHale, M. E. R.; Powell, J . R.; Kauppila, A.-S. M.; Acree, W. E., J r.; Huyskens, P. L. Thermochemical Investigations of HydrogenBonded Solutions. Part 11. Expressions for Predicting Anthracene Solubilities in Alcohol + Alkoxyal cohol Mixtures Based on Mobile Order Theory. J . Solution Chem. 1996a, 25, 1089-1104.

(7) McHale, M. E. R.; Zvaigzne, A. I.; Powell, J . R.; Kauppila, A.-S M.; Acree, W. E., J r. Thermochemical Investigations of HydrogenBonded Solutions. Part 9. Comparison of Mobile Order Theory and the Kretschmer-Wiebe Association Model for Predicting Pyrene Solubilities in Binary Alcohol + Alcohol Solvent Mixtures. Phys. Chem. Liq. 1996b, 32, 67-85.

Received for review December 2, 1996. Accepted J anuary 16 1997. ${ }^{\otimes}$

J E9603887

${ }^{\otimes}$ Abstract published in Advance ACS Abstracts, March 1, 1997. 